\title{
Synthesis and Spectroscopic
} Investigation of Aggregation Through Cooperative $\pi-\pi$ and $\mathrm{C}-\mathrm{H} \cdot \cdots \mathrm{O}$ Interactions in a Novel Pyrene Octaaldehyde Derivative

\author{
Gandikota Venkataramana and Sethuraman \\ Sankararaman* \\ Department of Chemistry, Indian Institute of Technology Madras, \\ Chennai - 600 036, India \\ sanka@iitm.ac.in
}

\section{Supporting Information}

Synthesis of 3: A mixture of 2 (0.45 g, $1.68 \mathrm{mmol}), \mathrm{Pd}\left(\mathrm{PPh}_{3}\right)_{4}(0.077 \mathrm{~g}, 0.067 \mathrm{mmol})$, $\mathrm{CuI}(0.025 \mathrm{~g}, 0.134 \mathrm{mmol})$ and $\mathrm{Et}_{3} \mathrm{~N}(8.0 \mathrm{ml})$ was stirred at room temperature for $15 \mathrm{~min}$ under $\mathrm{N}_{2}$ atmosphere. Compound $1(0.1 \mathrm{~g}, 0.34 \mathrm{mmol})$ was added and stirring was continued at $50{ }^{\circ} \mathrm{C}$ for $4 \mathrm{~h}$ upon which the reaction mixture turned dark red. Solvent was removed under reduced pressure and the crude product was purified by column chromatography on silica gel and eluted with a mixture of $\mathrm{CH}_{2} \mathrm{Cl}_{2}$ and hexane $(1: 1 \mathrm{v} / \mathrm{v})$ to yield octaaldehyde 3 as a dark red fluorescent solid (0.3 g, 85\%). Further recrystallization from a mixture of $\mathrm{CH}_{2} \mathrm{Cl}_{2}$ and hexane gave pure sample of 3. $\mathrm{mp}>240$ ${ }^{\circ} \mathrm{C}$ (decomp without melting), IR (KBr) 2963, 2855, 2741, 2194, 1692, $1384 \mathrm{~cm}^{-1} ;{ }^{1} \mathrm{H}$ NMR (CDCl $\left.3,400 \mathrm{MHz}, 25{ }^{\circ} \mathrm{C}, 4 \times 10^{-2} \mathrm{M}\right) \delta 10.64$ (s, 8H), 8.21 (s, 4H), 8.17 (s, 8H), 7.98 (s, 2H), 1.46 (s, 36H); ${ }^{13} \mathrm{C}$ NMR $\left(\mathrm{CDCl}_{3}, 100 \mathrm{MHz}, 25{ }^{\circ} \mathrm{C}\right) \delta 190.1,153.0,136.5$, 134.3, 131.6, 130.6, 127.1, 124.6, 122.4, 117.7, 99.5, 88.5, 35.3, 30.9; MALDI-TOF MS: 
Calcd for $\mathrm{C}_{72} \mathrm{H}_{58} \mathrm{O}_{8}$ 1050.4; found m/z (rel intensity) 1050.5 (100), 1051.5 (75), 1052.5 (30), $1053.6(8)$.

Fluorescence spectral measurements were made after degassing the solutions with nitrogen for 15 minutes.

Figure 1. ${ }^{1} \mathrm{H}$ NMR spectrum of 3 in $\mathrm{CDCl}_{3}(400 \mathrm{MHz})$ at $25{ }^{\circ} \mathrm{C}, 4 \times 10^{-2} \mathrm{M}$.

Figure 2. ${ }^{13} \mathrm{C}$ NMR spectrum of 3 in $\mathrm{CDCl}_{3}(100 \mathrm{MHz})$ at $25{ }^{\circ} \mathrm{C}, 4 \times 10^{-2} \mathrm{M}$.

Figure 3. Temperature dependent ${ }^{1} \mathrm{H}$ NMR spectra of 3 in $\mathrm{CDCl}_{3}(400 \mathrm{MHz}), 4 \times 10^{-}$ ${ }^{2} \mathrm{M}$.

Figure 4. Concentration dependent ${ }^{1} \mathrm{H}$ NMR spectra of 3 in $\mathrm{CDCl}_{3}(400 \mathrm{MHz})$. Spectrum A: $4 \times 10^{-2} \mathrm{M}$, spectrum B: $8 \times 10^{-3} \mathrm{M}$ at $25{ }^{\circ} \mathrm{C}$.

Figure 5. Color of 3 in $\mathrm{CDCl}_{3}$ in various concentrations. A: $4 \times 10^{-2} \mathrm{M}, \mathbf{B}: 8 \times 10^{-3}$ M, C: $1 \times 10^{-5} \mathrm{M}$. 


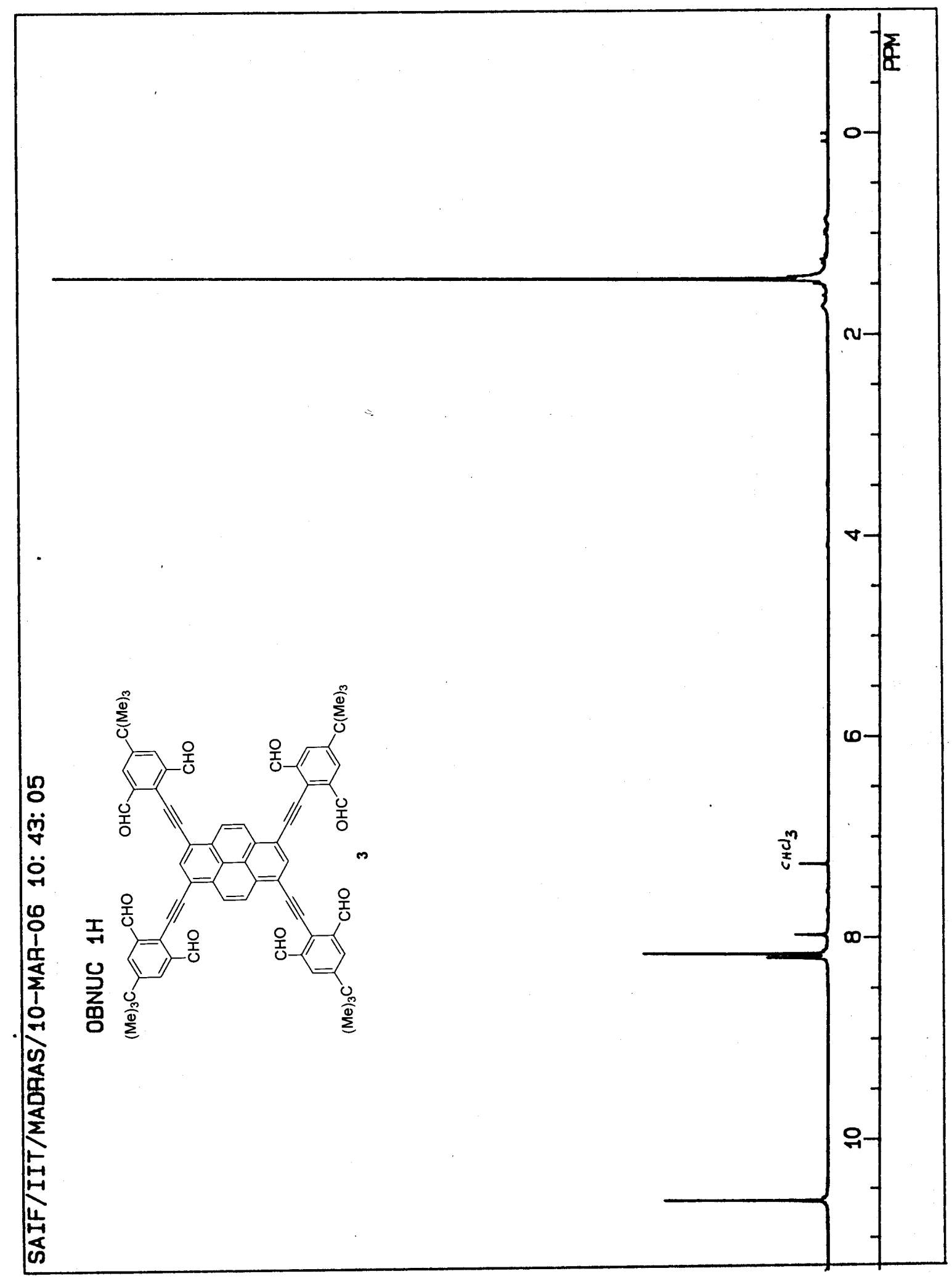

Figure 1. ${ }^{1} \mathrm{H}$ NMR spectrum of 3 in $\mathrm{CDCl}_{3}(400 \mathrm{MHz})$ at $25{ }^{\circ} \mathrm{C}, 4 \times 10^{-2} \mathrm{M}$. 


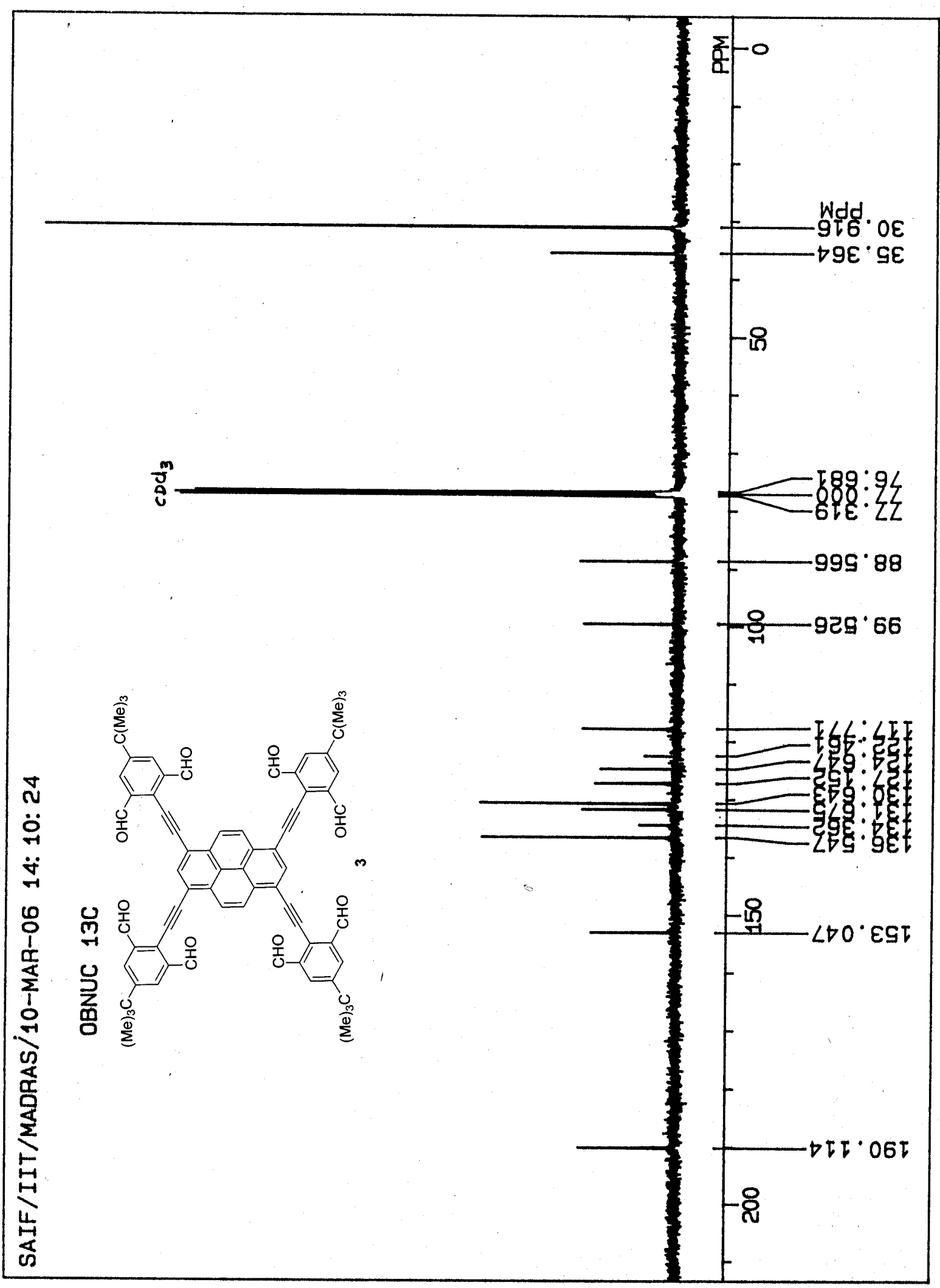

Figure 2. ${ }^{13} \mathrm{C}$ NMR spectrum of 3 in $\mathrm{CDCl}_{3}(100 \mathrm{MHz})$ at $25{ }^{\circ} \mathrm{C}, 4 \times 10^{-2} \mathrm{M}$. 

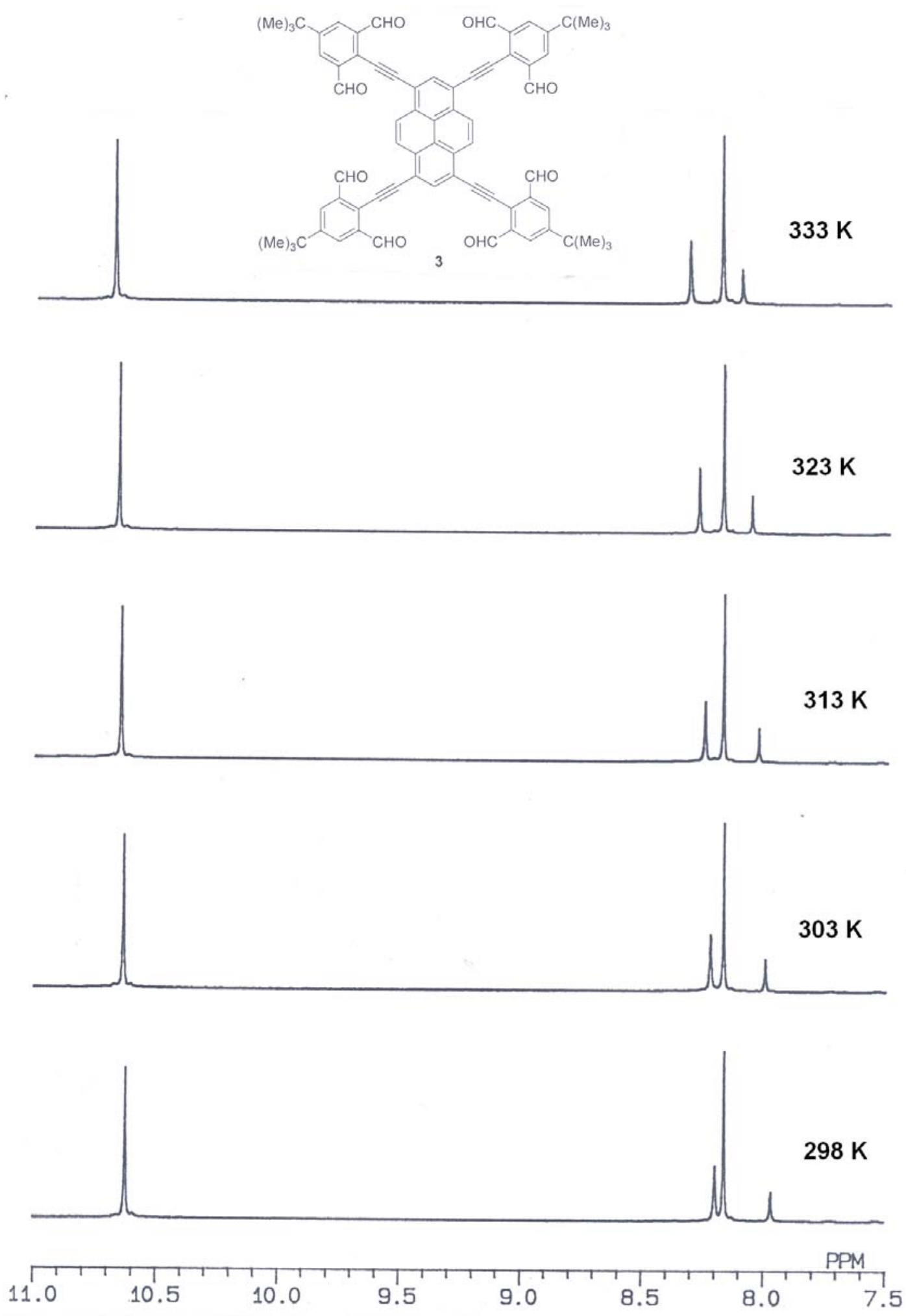

Figure 3. Temperature dependent ${ }^{1} \mathrm{H}$ NMR spectra of 3 in $\mathrm{CDCl}_{3}(400 \mathrm{MHz}), 4 \times 10^{-2}$ M. 


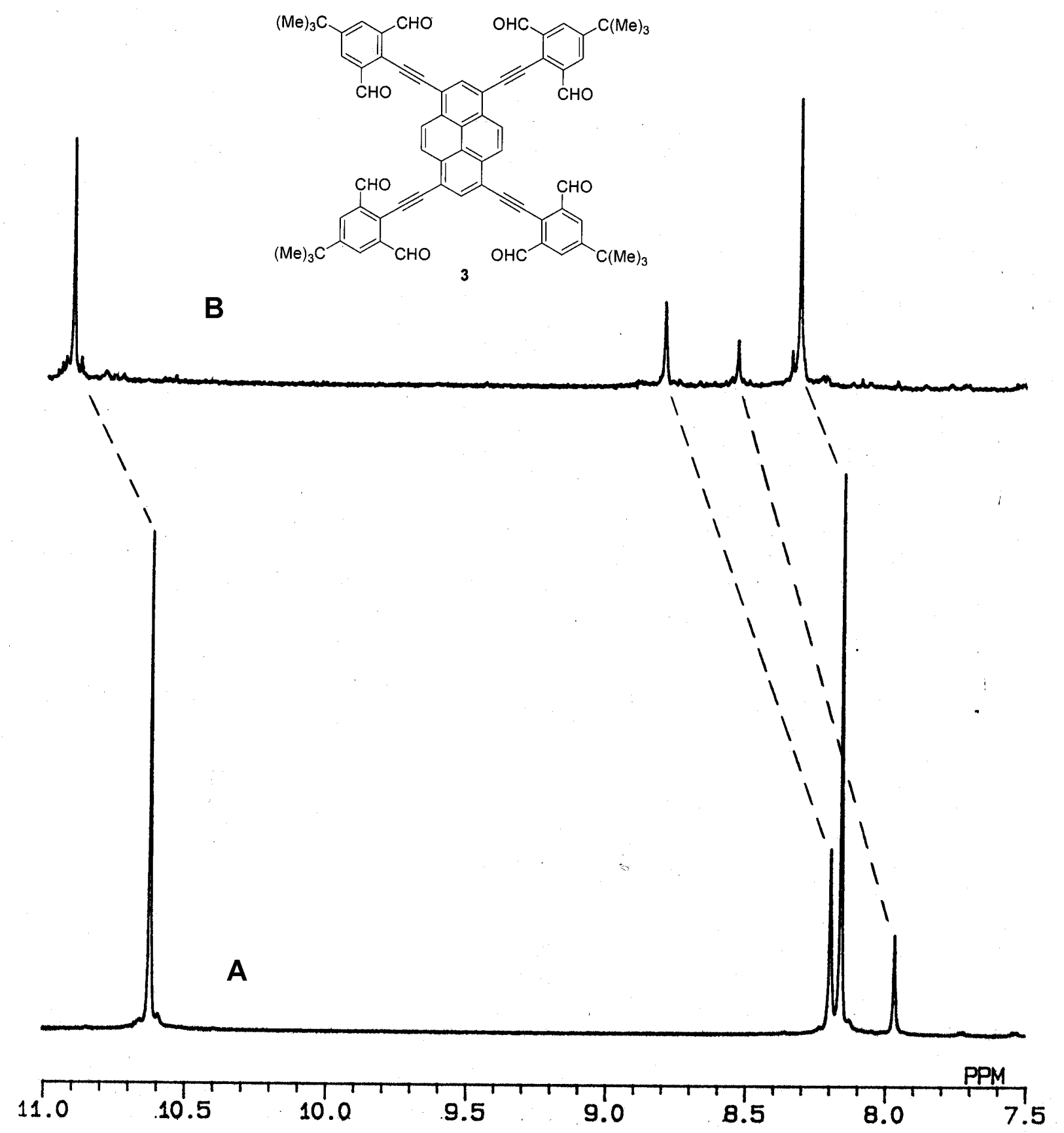

Figure 4. Concentration dependent ${ }^{1} \mathrm{H}$ NMR spectra of 3 in $\mathrm{CDCl}_{3}(400 \mathrm{MHz})$.

Spectrum A: $4 \times 10^{-2} \mathrm{M}$, spectrum B: $8 \times 10^{-3} \mathrm{M}$ at $25{ }^{\circ} \mathrm{C}$. 


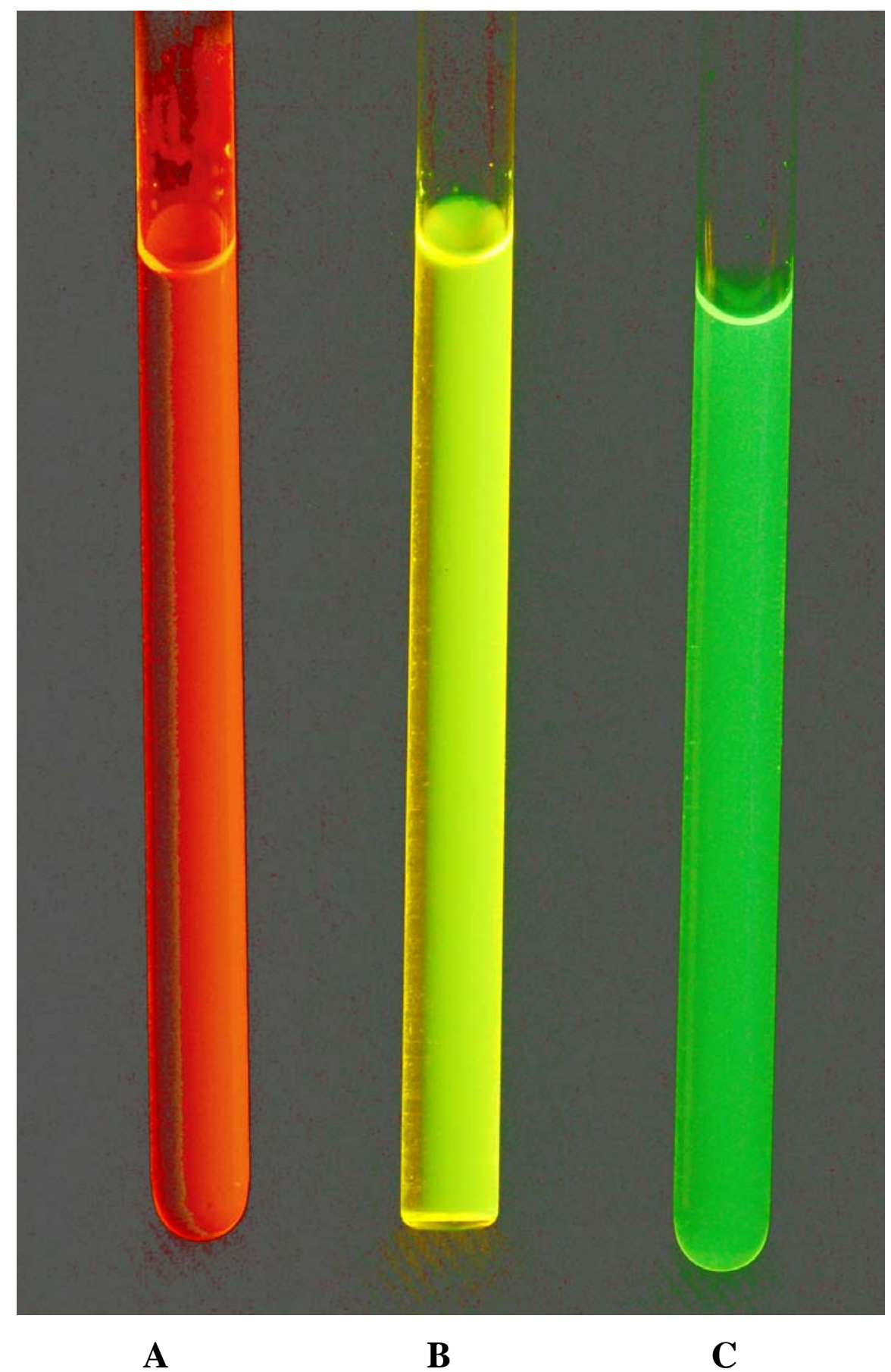

Figure 5. Color of 3 in $\mathrm{CDCl}_{3}$ in various concentrations. A: $4 \times 10^{-2} \mathrm{M}, \mathbf{B}: 8 \times 10^{-3}$ M, C: $1 \times 10^{-5} \mathrm{M}$ 